\title{
Cytomegalovirus retinitis mimicking intraocular lymphoma
}

\author{
Patrick Gooi' \\ James Farmer ${ }^{2}$ \\ Bernard Hurley ${ }^{3}$ \\ Elliott Brodbaker ${ }^{4}$ \\ 'Department of Ophthalmology, \\ University of Calgary, Calgary, \\ Alberta, Canada; ${ }^{2}$ Department \\ of Pathology and Lab Medicine \\ University of Ottawa and The Ottawa \\ Hospital, Ottawa, Ontario, Canada; \\ ${ }^{3}$ Department of Ophthalmology, \\ University of Ottawa Eye Institute \\ and The Ottawa Hospital, Ottawa, \\ Ontario, Canada; ${ }^{4}$ Faculty of Medicine, \\ University of Ottawa, Ottawa, \\ Ontario, Canada
}

\begin{abstract}
We present a case of an unusual retinal infiltrate requiring retinal biopsy for definitive diagnosis. A 62-year-old man with treated lymphoma presented with decreased vision in the right eye associated with a white retinal lesion, which extended inferonasally from an edematous disc. Intraocular lymphoma was considered as a diagnosis; thus, the patient was managed with vitrectomy and retinal biopsy. Cytological analysis of the vitreous aspirate could not rule out a lymphoproliferative disorder. The microbial analysis was negative. Histology of the lesion showed extensive necrosis and large cells with prominent nucleoli. To rule out lymphoma, a battery of immunostains was performed and all were negative. However the limited amount of tissue was exhausted in the process. Subsequently, a hematoxylin and eosin (H/E) slide was destained, on which a CMV immunostain was performed. This revealed positivity in the nuclei and intranuclear inclusions within the large atypical cells. A diagnosis of CMV retinitis was made. Retinal biopsy may provide a definitive diagnosis and direct patient care toward intravenous gancyclovir in the case of CMV or toward radiation and chemotherapy for intraocular lymphoma. When faced with a limited amount of tissue, destaining regular $\mathrm{H} / \mathrm{E}$ slides is a possible avenue to performing additional immunohistochemical studies.
\end{abstract}

Keywords: CMV retinitis, retinal biopsy, immunohistochemistry, destaining

\section{Introduction}

The etiology of retinal and choroidal lesions can often be clinically determined with the aid of ancillary tests such as ultrasound and fluorescein angiography. However, there are instances where either an infective or malignant lesion is in question, and a retinal biopsy is warranted to provide a definitive diagnosis (Johnston et al 2004). We present a case of an immuncompromised individual with an unusual retinal infiltrate requiring retinal biopsy for definitive diagnosis.

\section{Case report}

A 62-year-old man was assessed by the retinal service of the Ottawa Eye Institute on July 19, 2006 for decreased vision in the right eye associated with a white retinal lesion. His past medical history included follicular lymphoma (a form of non-Hodgkin lymphoma), diagnosed 6 years previously and treated with chemotherapy. 5 months prior to presentation, he had been admitted for biopsy-proven CMV esophagitis. On examination, the right eye had slightly decreased visual acuity (20/40), and substantial inflammation was present in the anterior and posterior segments. Fundoscopy revealed a whitish retinal infiltrate extending inferonasally from an edematous disc (Figure 1). Fluorescein angiography showed a pattern of early hypofluorescence and late hyperfluorescence consistent with an inflammatory lesion. Intraocular lymphoma was considered as a diagnosis; thus, the patient was managed with vitrectomy and retinal biopsy. Given the location of the lesion, we felt it would be more definitive to receive a direct tissue sample rather than relying on overlying vitreous cells which may not truly reflect the nature of the lesion. 


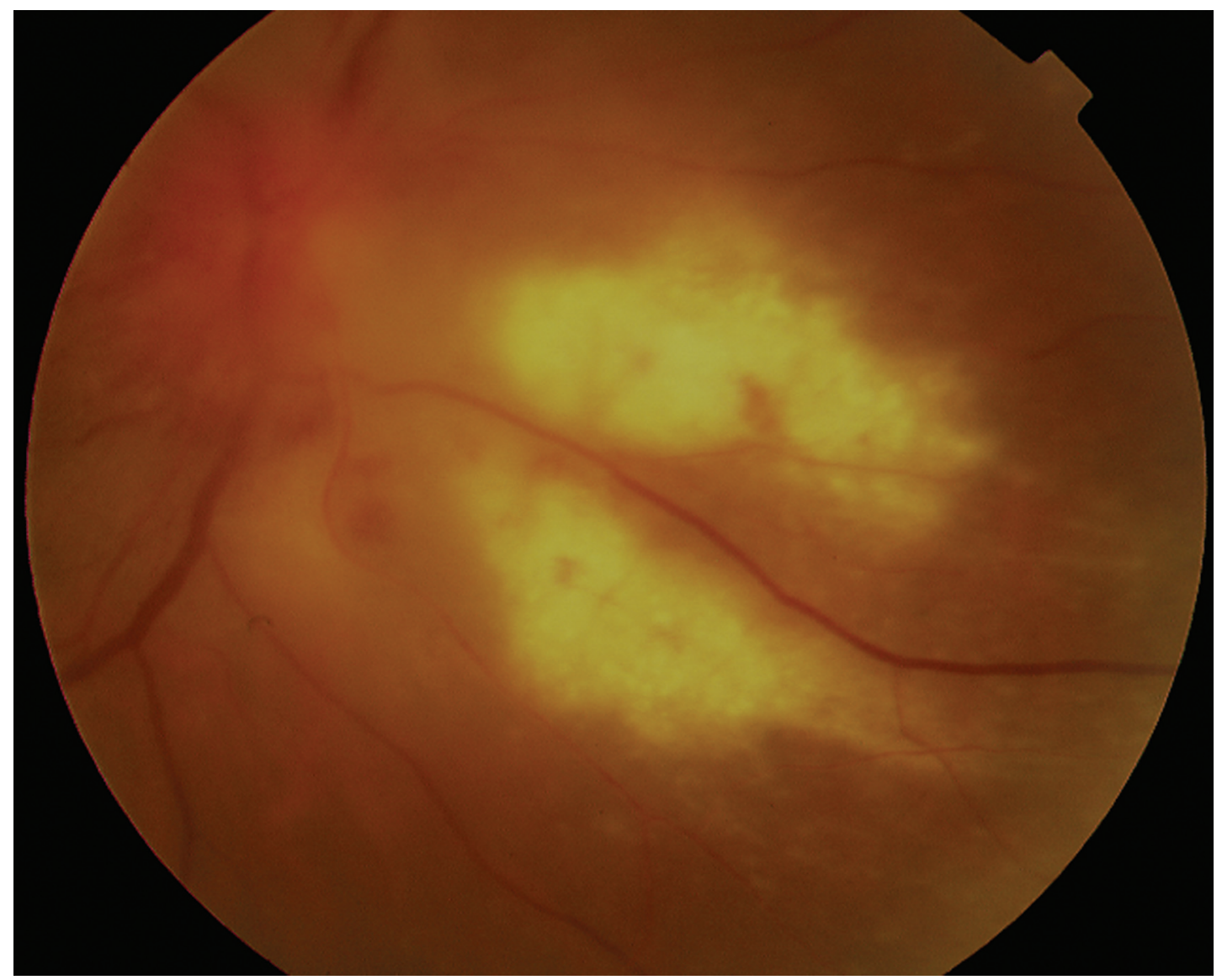

Figure I Right fundus photograph illustrating a large, dense, whitish retinal lesion extending inferonasally from the disc with some intraretinal hemorrhage surrounding the lesion.

Cytological analysis of the vitreous aspirate could not rule out a lymphoproliferative disorder. Histology of the lesion showed extensive necrosis and large cells with prominent nucleoli. To exclude lymphoma, a battery of immunostains was performed and all were negative. The limited amount of tissue was exhausted in the process. Some atypical cells appeared to contain large intranuclear inclusions with a surrounding clear halo (Figure 2), which raised the suspicion of a cytomegalovirus (CMV) infection. The specimen was reviewed with neuropathology and lymphoma specialists who felt the findings were not entirely characteristic for CMV. However, the microbial analysis, including CMV and Herpes viral cultures, was negative. Furthermore, the tissue from the retinal biopsy was exhausted, which limited avenues in pursuing a CMV retinitis diagnosis.

Rather than risk another vitreous biopsy, a hematoxylin and eosin (H/E) slide was destained, on which a CMV immunostain was performed. This revealed positivity in the nuclei and intranuclear inclusions within the large atypical cells (Figure 2). A diagnosis of CMV retinitis was made.
At 3 months post-vitrectomy, the patient showed improvement in vision and regression of the retinal lesion. As a result of the findings, he did not undergo further treatment for his lymphoma.

\section{Discussion}

Retinitis resulting from CMV infection is often felt to mostly affect individuals with impaired lymphocyte counts (Vote et al 2005). CMV retinitis in the setting of lymphoma often manifests as retinal whitening with hemorrhage and vasculitis (Chawla et al 2005). This clinical constellation can encompass a fairly wide differential including lymphoma, viral infections (CMV, herpes simplex, varicella zoster) (Chawla et al 2005) and other infectious agents (Toxoplasma gondii, Treponema pallidum) (Johnston et al 2004; Chawla et al 2005).

Although CMV infection is fairly common in patients with malignant lymphoma, with 13.6 to $74 \%$ (Chawla et al 2005) of patients showing evidence of infection on autopsy, CMV retinitis in the setting of malignant lymphoma is quite rare, with 11 cases previously reported (Akpek et al 1999; Chawla et al 2005; Vote et al 2005). Most of the cases 


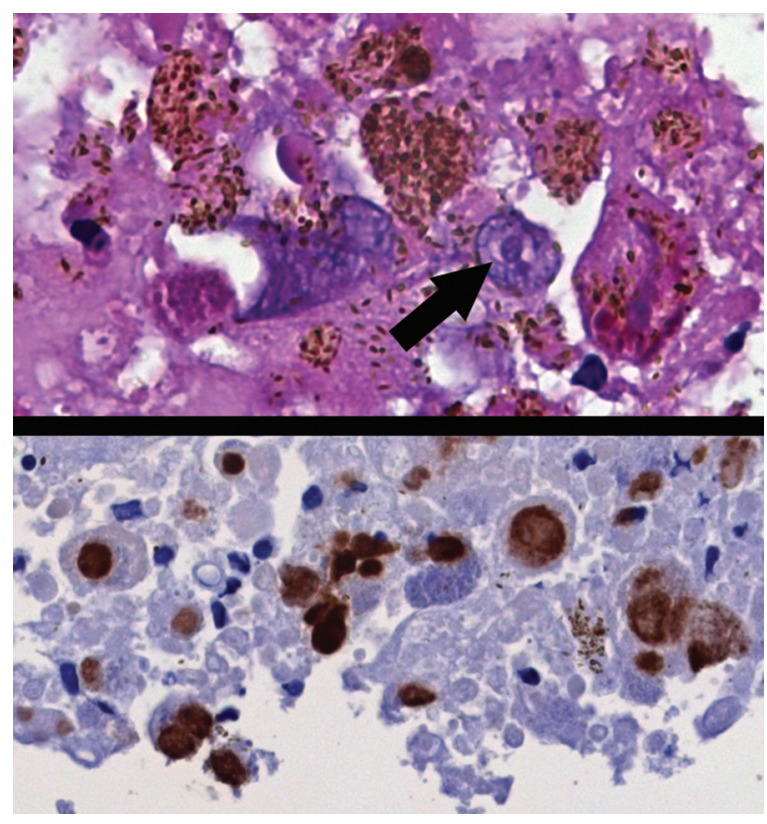

Figure 2 Top: Cell containing an intranuclear inclusion with a surrounding clear halo (arrow) suggestive of CMV infection (original magnification 640x). Bottom: CMV immunohistochemical stain of the retinal biopsy showing positivity in the nuclei and intranuclear inclusions (original magnification 640x).

presented with a white retinal lesion (6/11) with intraretinal hemorrhages (8/11). Vasculitis (3/11) and sheathing (3/11) were reported less often. Vitritis (3/11) and an anterior chamber reaction (2/11) were also less common. Only 3 cases were bilateral.

This case illustrates that retinal biopsy may provide a definitive diagnosis and direct patient care toward intravenous gancyclovir in the case of CMV (Vote et al 2005) or toward radiation and chemotherapy for intraocular lymphoma (Johnston et al 2004). Retinal lesions large enough for a biopsy are rarely encountered and often furnish very little tissue. When faced with a limited amount of tissue, destaining H/E slides is a possible avenue to performing additional immunohistochemical studies. In this case, the CMV immunohistochemical studies were essential for the diagnosis, as the neuropathology specialists were not convinced of the presence of CMV infection in the original $\mathrm{H} / \mathrm{E}$ sections. Alternatively, if no tissue is available, PCR studies on an aqueous humor sample or vitreous aspirate can show the presence of $\mathrm{CMV}$, which has excellent sensitivity (92.3\%) and specificity (98\%) (Chawla et al 2005). These studies should be specifically ordered in the workup of unknown retinal lesions of a possible uveitic etiology in immunocompromised patients.

\section{Disclosures}

The authors have no proprietary interests in any aspect of this study.

\section{References}

Akpek EK, Kent C, Jakobiec F, et al. 1999. Bilateral acute necrosis caused by Cytomegalovirus in an immunocompromised patient. Am J Ophthalmol, 127:93-5.

Chawla R, Venkatesh P, Garg SP, et al. 2005. Cytomegalovirus retinitis in a patient with non-Hodgkin's lymphoma: a diagnostic dilemma. Eur J Ophthalmol, 15:153-7.

Johnston RL, Tufail A, Lightman S, et al. 2004. Retinal and choroidal biopsies are helpful in unclear uveitis of suspected infectious or malignant origin. Ophthalmology, 111:522-8.

Vote B, Russell M, Polkinghorne P, et al. 2005. Recurrent cytomegalovirus retinitis in a patient with a normal lymphocyte count who had undergone splenectomy for lymphoma. Retina, 25:220-21. 
